\title{
Teaching and Learning of Number Systems and Arithmetic (Focusing Especially on Primary Education)
}

\author{
Joana Brocardo and Geoffrey B. Saxe
}

\section{Aims, Themes and Organization}

\section{Aims and Themes}

The group's focus is on individuals' elementary mathematical representations and understandings with a special interest in the way these aspects of cognition develop through activities in and out of school. The mathematical domains of concern include whole numbers, integers, and rational numbers as well as representations related to each of these domains.

A related interest of the group is socio-cultural analyses. These analyses would include the ways that mathematics (including mathematical argumentation, representations, problem solving, teaching-learning interactions) is constituted in everyday practices as well as the interplay between developing mathematical understanding and representations in and out of school.

The group encourages cross-disciplinary contributions, including (but not limited to) participation by educational researchers, mathematics educators, developmental psychologists, and cultural anthropologists.

Organizers Co-chairs: Joana Brocardo (Portugal), Geoffrey B. Saxe (USA); Team Members: Maria Lucia Faria Moro (Brazil), mlfmoro@sul.com.br, Minkyung Kim (Korea)

mkkim@ewha.ac.kr; Liaison IPC member: K. Subramaniam.

\footnotetext{
J. Brocardo ( $\square)$

Setúbal, Portugal

e-mail: joana.brocardo@ese.ips.pt

G.B. Saxe

Berkeley, USA

e-mail: saxe@berkeley.edu

(C) The Author(s) 2015

S.J. Cho (ed.), The Proceedings of the 12th International Congress

on Mathematical Education, DOI 10.1007/978-3-319-12688-3_32
} 


\section{Organization}

TSG 7 received 29 submissions. We decided to emphasize discussion, articulating oral presentation and its discussion with poster presentations.

Two members of the organizing team and one external reviewer reviewed each paper. From the reviews and interactions by email among the members of the Organizing Team, an agreement was reached on a final list of presentations and posters, leading to 10 oral presentations and 17 posters. Due to cancellations only 10 posters were presented in two slots with 5 in each one. This turned the poster sessions of the group into an interactive session, in which each poster was presented by the author(s) and then discussed with all the participants.

The participants in the group came from 15 different countries of North and South America, Asia, Africa and Europe.

\section{Papers, Posters, and Discussion Topics that Emerged in the Sessions}

The presentations and the discussion varied markedly, reflecting diverse orientations and focal interests in teaching and learning about number systems and operations. Though diverse, the papers and posters conformed to four general themes.

The first theme was formalization of mathematical ideas, mathematical contexts, and models. The presentations and the discussion highlighted potentialities and barriers to the learning and teaching of number system and operations.

The second theme engaged participants with elementary mathematical representations and understandings that individuals construct. The presentations included case studies that illustrate the development of representation and understandings through activities in and out of school.

A third concerned kinds of numbers that are the focus of teaching and learning. These papers focused on teaching and learning of whole, fractional and decimal numbers. Papers and posters presented and analyzed processes whereby students overcome their misunderstandings and difficulties.

Finally the group discussed examples of everyday practices in school that can promote understanding in the domain of number and operations as well as the interplay between developing mathematical understanding and representations in and out of school. This discussion included examples and ideas related with mathematical argumentation, representations, problem solving and teaching-learning interactions.

The schematic contained in Fig. 1 illustrates the principal focus of TSG7 on number systems and operations themes, the concern for understanding processes of teaching and learning related to the focus, and the paper presentations, posters, and discussion that emerged on the four themes. 


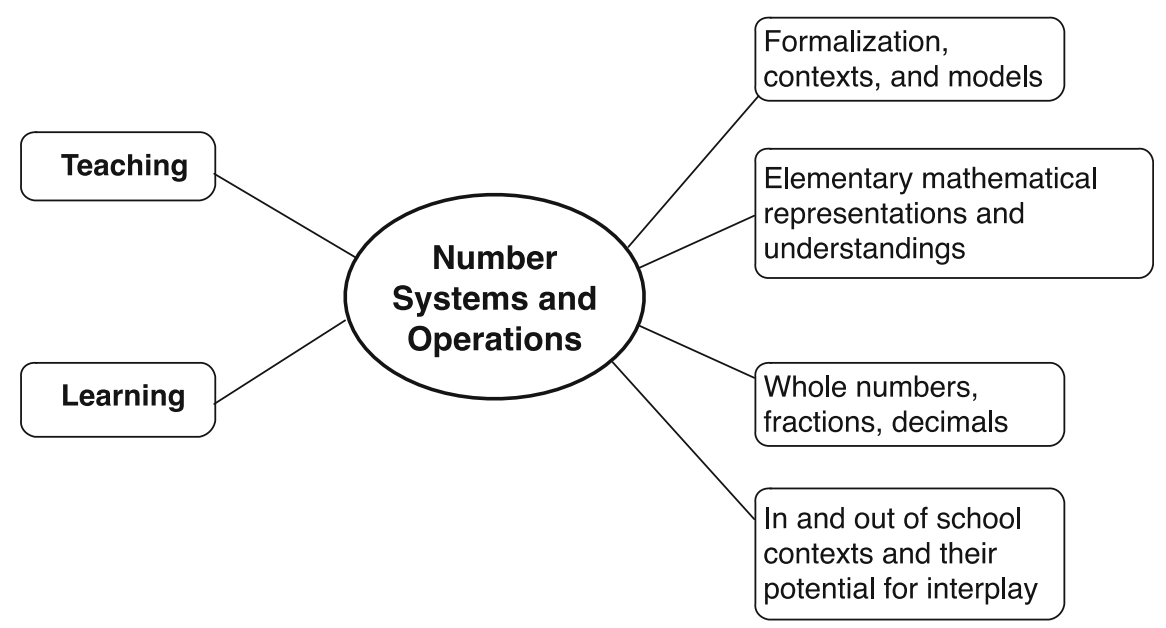

Fig. 1 Schematic of themes and presentation topics in TSG 7 for the 2012 meetings

Open Access This chapter is distributed under the terms of the Creative Commons Attribution Noncommercial License, which permits any noncommercial use, distribution, and reproduction in any medium, provided the original author(s) and source are credited. 